\title{
EFECTIVIDAD EN EL DIAGNÓSTICO DE CÁNCER DE PRÓSTATA DE DOS ESQUEMAS DE BIOPSIA TRANSRECTAL ECODIRIGIDA. ESTUDIO ALETORIZADO
}

\author{
Felipe Herranz Amo, José Jara Rascón, María José Cancho Gil, Juan Tabares Jiménez, Roberto \\ Molina Escudero y Carlos Hernández Fernández.
}

Servicio de Urología. Hospital General Universitario Gregorio Marañón. Madrid. España.

\begin{abstract}
Resumen.- OBJETIVO: Evaluar si un esquema de biopsia de próstata de 10 cilindros es superior al esquema de 6 cilindros en el diagnóstico de cáncer de próstata, en pacientes que van a ser sometidos a una primera biopsia, con un tacto rectal no sospechoso y un PSA entre 3,5 y $20 \mathrm{ng} / \mathrm{ml}$.

MÉTODO: Estudio prospectivo aleatorizado entre 2 esquemas de biopsia de próstata con estratificación de la aleatorización por volumen prostático ( $\leq 50$ cc y > 50 cc.). Mediante predeterminación del tamaño muestral se obtuvo un mínimo de 304 pacientes para poder responder al objetivo principal. El análisis estadístico
\end{abstract}

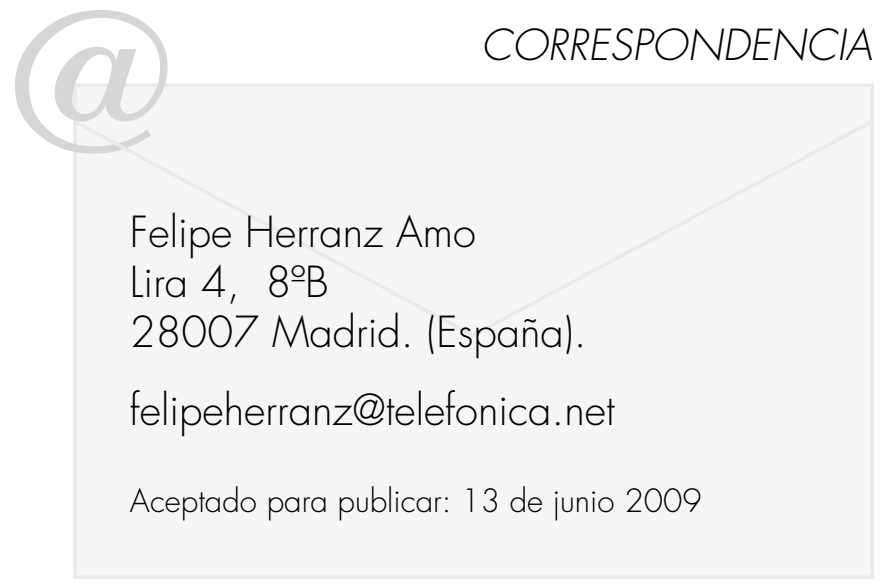

se realizó por "intención de tratar", mediante el test de la Chi cuadrado y análisis uni y multivariante mediante regresión logística.

RESULTADOS: Se detectó cáncer en el 27,3\% de los casos. Existió una relación significativa entre el diagnóstico de cáncer y la edad ( $p=0,03)$, el volumen prostático ( $p=0,0001)$ y la existencia de nódulo ecográfico $(p=0,0001)$. No se observó relación con el número de cilindros en el total de la serie $(p=0,37)$, ni en la próstatas $\leq 50 \mathrm{cc}(p=0,87)$ ni en las $>50 \mathrm{cc}(p=0,09)$. En el análisis multivariante la edad $>70$ años $(p=0,005)$, el volumen de la próstata $\leq 50 \mathrm{cc}(\mathrm{p}=0,001)$ y la existencia del nódulo ecográfico $(p=0,003)$ se identificaron como variables independientes asociadas al diagnóstico de cáncer.

CONCLUSIÓN: No hemos encontrado diferencias estadísticamente significativas entre ambos esquemas de biopsia de próstata. En las glándulas mayores de 50 cc. el esquema de 6 cilindros puede ser insuficiente.

Palabras clave: Cáncer de próstata. Diagnóstico. Biopsia transrectal ecodirigida. Estudio aleatorizado.

Summary.- OBJECTIVE: To determine whether a 10core prostate biopsy scheme is superior to the sextant scheme in diagnosing prostate cancer in patients programmed for a first biopsy, with negative rectal digital examination and PSA between $3.5-20 \mathrm{ng} / \mathrm{ml}$.

METHODS: A randomized prospective study was performed comparing two prostate biopsy schemes, with randomization stratification according to prostate volume ( $\leq 50 \mathrm{ml}$ and > $50 \mathrm{mll}$. Sample size predetermination yielded a minimum of 304 patients in order to achieve the primary objective. Statistical analysis was carried out 
on an intent-to-treat basis, using the chi-squared test and uni- and multivariate analysis via logistic regression.

RESULTS: Cancer was detected in $27.3 \%$ of the cases. A significant association was observed between cancer diagnosis and age $(p=0.03)$, prostate volume $(p=0.0001)$ and ultrasound nodule identification $(p=0.0001)$. No correlation was observed with the total number of cores in the series $(p=0.37)$ or with prostate volume $\leq 50 \mathrm{ml}(p=0.87)$ or $>50 \mathrm{ml}(p=0.09)$. In the multivariate analysis, age $>70$ years $(p=0.005)$, prostate volume $\leq 50 \mathrm{ml}(p=0.001)$, and ultrasound nodule identification ( $p=0.003$ ) were identified as independent variables associated to cancer diagnosis.

CONCLUSION: No statistically significant differences were found between the two prostate biopsy schemes. In glands over $50 \mathrm{ml}$ in size, the sextant scheme may prove to be insufficient.

Keywords: Prostate cancer. Diagnosis. Ultrasound-guided transrectal prostate biopsy. Randomized study.

\section{INTRODUCCIÓN}

La biopsia transrectal ecodirigida de la próstata se considera la técnica de elección para el diagnóstico del cáncer de la glándula prostática (1). La biopsia sextante de Hodge (2), aún con la modificación de Stamey tiene entre un 20 y un $30 \%$ de falsos negativos (4-6). Numerosos autores han intentado diseñar el esquema de biopsia "ideal", en el cual se obtuviera el mayor rendimiento posible con el menor numero de punciones, casi siempre basándose en estudios no aleatorizados (7).

Varios autores (8-10) encontraron una relación entre el rendimiento de la biopsia sextante clásica y el volumen de la glándula prostática, disminuyendo con esta técnica de forma importante la detección de cáncer en las próstatas más grandes. Establecieron en los $50 \mathrm{cc}$. de volumen glandular el punto de corte, a partir del cuál la biopsia sextante es insuficiente y por lo tanto se debería de incrementar el número de cilindros.

La EAU (1) recomienda, basándose en estudios clínicos no aleatorizados (recomendación B), la realización de un mínimo de 10 cilindros por paciente, aumentando el número en las glándulas más grandes.

Presentamos un estudio aleatorizado en el que comparamos dos esquemas de biopsia transrectal de la próstata. El objetivo principal de este es- tudio fue evaluar si un esquema de 10 cilindros es superior al esquema de 6 cilindros en el diagnóstico de cáncer de próstata en los pacientes que van a ser sometidos a una primera biopsia, con un tacto rectal no sospechoso y con unos niveles de PSA entre 3,5 y $20 \mathrm{ng} / \mathrm{ml}$.

\section{MATERIAL Y MÉTODO}

\section{- Población diana:}

El estudio de realizó en el Servicio de Urología del H.G.U. Gregorio Marañón, en el periodo de tiempo comprendido entre Mayo del 2006 y Noviembre del 2007. La población diana fueron los pacientes del Área 1 de la Comunidad de Madrid procedentes de la consulta externa del mismo Hospital o de las consultas de los Centros de Especialidades con sospecha de cáncer de próstata por elevación del PSA.

\section{- Diseño del estudio:}

Estudio prospectivo aleatorizado, unicéntrico entre dos esquemas de biopsia transrectal ecodirigida (6 o 10 cilindros) con estratificación de la aleatorización en función del volumen prostático $\leq 50 \mathrm{cc}$. y $>50$ cc.), debido a que el volumen prostático puede suponer un sesgo en el estudio. La aleatorización se realizó según dos tablas de aleatorización generadas por ordenador, una para próstatas menores o iguales de $50 \mathrm{cc}$. y otra para las mayores de $50 \mathrm{cc}$.

\section{- Criterios de inclusión y exclusión:}

Para su inclusión en el estudio los sujetos debían de cumplir todos los criterios siguientes:

o Pacientes mayores de 18 años.

- No sometidos nunca a una biopsia transrectal de próstata.

- PSA entre 3,5 y $20 \mathrm{ng} / \mathrm{ml}$.

- Tacto rectal no sospechoso de cáncer de próstata.

- Información y aceptación del paciente mediante la firma del Documento de Consentimiento Informado para participar en el estudio.

Los criterios de exclusión del estudio fueron lo siguientes:

- Antecedentes de prostatitis aguda o infección del tracto urinario en los 3 meses previos a la biopsia.

- Infección del tracto urinario activa.

o Portador de sonda vesical.

- Tratamiento con análogos de la LH-RH, antiandrógenos o inhibidores de la 5-a-reductasa.

- Pacientes con antecedentes de resección transuretral de próstata o adenomectomía. 


\section{- Descripción de los dos esquemas de biopsia} (Figura 1):

o Esquema de 6 cilindros: Se realizaron 3 biopsias periféricas de cada lóbulo prostático según la Técnica de Stamey.

- Esquema de 10 cilindros: Los anteriores más 2 cilindros mediales de cada lóbulo.

\section{- Rutina del estudio:}

o Confirmación de los criterios de inclusión y exclusión.

o Firma del Documento de Consentimiento Informado.

o Realización del tacto rectal.

- Medición ecográfica del volumen prostático.

- Consulta de la tabla de aleatorización correspondiente y comprobación del esquema de biopsia asignado (6 o 10 cilindros).

- Orden de realización de las biopsias y etiquetado:

1ํ - 3 biopsias periféricas del lóbulo derecho - envase $\mathrm{n}^{\circ} 1$

2ㅇ - 3 biopsias periféricas del lóbulo izquierdo - envase $\mathrm{n}^{\circ} 2$

3 - 2 biopsias mediales del lóbulo derecho. - envase $\mathrm{n}^{\circ} 3$.

$4^{\circ}$ - 2 biopsias mediales del lóbulo izquierdo - envase $n^{\circ} 4$.

- Cumplimentación de la hoja de protocolo.

- Suministro al paciente una escala analógica visual de dolor para que la rellene en ese momento.

- Introducción de las variables en una base de datos creada en SPSS.

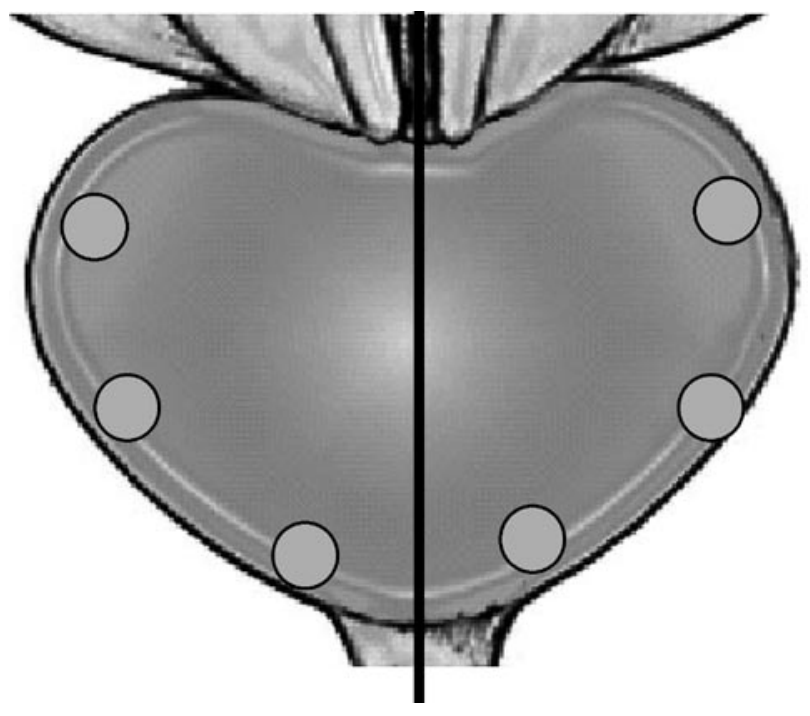

Esquema de 6 cilindros
- Recogida del informe de Anatomía Patológica e introducción de las variables en la base de datos.

\section{- Predeterminación del tamaño muestral:}

Asumiendo un error alfa $=0,05$ y uno beta $=$ 0,20 , para un contraste bilateral, unas pérdidas del $10 \%$, asumiendo una proporción de diagnóstico de 0,2 en uno de los esquemas de biopsia y esperando encontrar unas diferencias entre ambos esquemas de biopsia de 0,15, necesitaríamos 304 pacientes para que el estudio pueda responder al objetivo principal planteado.

\section{- Realización de la biopsia de la próstata:}

A los pacientes en el momento de la cita se les suministró una hoja de preparación previa a la biopsia mediante enemas de limpieza y profilaxis antibiótica con ciprofloxacino $500 \mathrm{mg}$. para tomar 2 horas antes del procedimiento y a las 12 horas de la toma anterior. Esta preparación es la que se realiza en la práctica clínica habitual en nuestro Servicio.

El procedimiento se realizó con un ecógrafo Brüel \& Kiaer modelo 3535 con un transductor transrectal multiplanar con una frecuencia de trabajo entre 6 y $10 \mathrm{Mhz}$., con canal de biopsia integrada tipo 8551. Las biopsias fueron realizadas por 3 staff del Servicio. Todos los miembros del equipo investigador tienen amplia experiencia en el manejo de este equipo y en la realización de biopsias transrectales ecodirigidas. Las biopsias de próstata se procesaron según el procedimiento habitual establecido entre los Servicios de Anatomía Patológica y Urología.

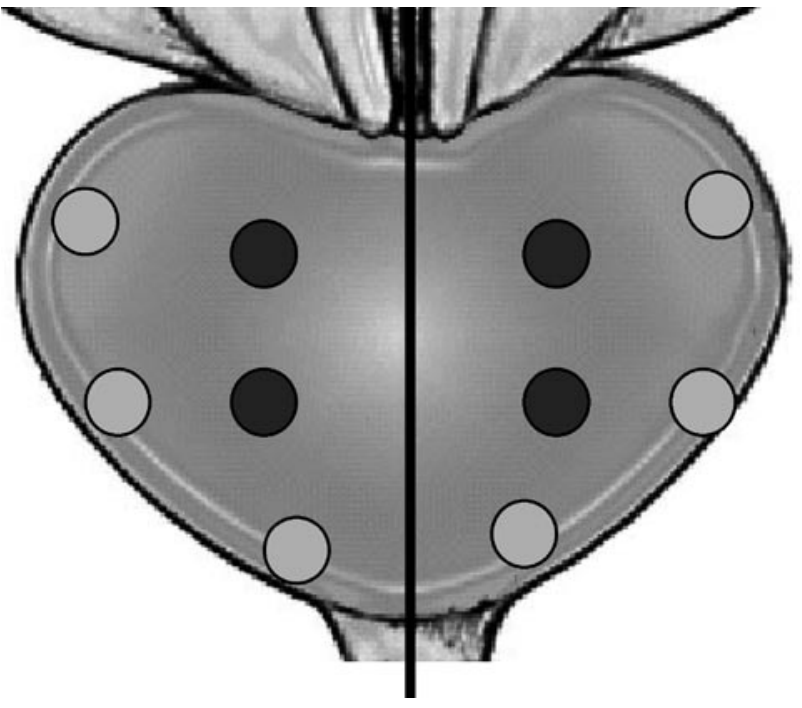

Esquema de 10 cilindros

FIGURA 1. Situación de las punciones en ambos esquemas de biopsia. 
TABLA I. CARACTERISTICAS CLÍNICAS DE LOS PACIENTES DEL ESTUDIO.

\begin{tabular}{|c|l|}
\hline Variable & Casos (\%) \\
\hline Edad en años: & $9(2,9 \%)$ \\
$\bullet<50$ & $71(22,5 \%)$ \\
$\bullet 51-60$ & $134(42,5 \%)$ \\
$\bullet 61-70$ & $96(30,5 \%)$ \\
$\bullet 71-80$ & $5(1,6 \%)$ \\
$\bullet>80$ & \\
$\bullet$ PSA en ng/ml: & $270(85,7 \%)$ \\
$\bullet>10$ & $45(14,3 \%)$ \\
$\bullet \leq 25$ & \\
$\bullet 26-50$ & $26(8,3 \%)$ \\
$\bullet 51-75$ & $152(48,3 \%)$ \\
$\bullet 75$ & $95(30,2 \%)$ \\
\hline Volumen prostático (cc.): & $42(13,3 \%)$ \\
\hline Nódulo prostático ecográfico: & \\
\hline & \\
\hline &
\end{tabular}

\section{- Confidencialidad de los datos:}

Después de la recogida de los datos del informe anatomopatológico, se procedió a la anonimización del registro de la base de datos, para cumplir con la Ley Orgánica 15/1999, de Protección de Datos de Carácter Personal (BOE, núm. 298, de 14 de diciembre de 1999).
- Análisis estadístico:

El análisis de la serie se realizó "por intención de tratar". Se ha realizado un análisis descriptivo de las variables analizadas mediante los estadísticos habituales para variables cuantitativas (media, desviación estándar, mediana, máximo y mínimo) y cualitativas (frecuencias absolutas y relativas). La existencia de cáncer de próstata (variable principal) y otras variables categóricas se compararon en ambas series de biopsia mediante el test de la Chi cuadrado. Se realizó un análisis global y estratificado por volumen prostático y niveles de PSA ( 10 o entre 10 y $20 \mathrm{ng} / \mathrm{ml}$.). Se realizó un análisis univariante y multivariante mediante regresión logística. La significación estadística se consideró cuando existió una $p<0,05$. Todos los cálculos se realizaron con el programa estadístico SPSS versión 15.0.

\section{- Seguimiento de la serie:}

Fuera de protocolo se realizó un seguimiento para conocer el tratamiento aplicado a los pacientes diagnosticados de cáncer de próstata $y$, la existencia de rebiopsia y su resultado en los pacientes con diagnóstico de patología benigna en la biopsia del estudio. Este seguimiento se cerró en el mes de abril del año 2009.

\section{- Autorización:}

Este estudio fue autorizado por el Comité de Ética e Investigación Clínica (CEIC) del Área 1 de la Comunidad de Madrid.

\section{RESULTADOS}

Se incluyeron 315 pacientes con una edad media de 65,8 $\pm 7,8(45-84)$ años y una mediana de 66 años, el PSA medio fue de $7,4 \pm 2,8(3,6-20)$ $\mathrm{ng} / \mathrm{ml}$, con una mediana de $6,7 \mathrm{ng} / \mathrm{ml}$ y el volumen prostático en cc. medido por ecografía fue de 50,3 $\pm 22,5$ (10-143) cc., con una mediana de 45,7 cc.

TABLA II. CARACTERÍSTICAS DE LOS PACIENTES SEGÚN LA ALEATORIZACIÓN A 6010 BIOPSIAS.

\begin{tabular}{|l|c|c|c|}
\hline Variable & 6 biopsias & 10 biopsias & p \\
\hline Edad (años) & $66 \pm 7,8$ & $66 \pm 8$ & 0,91 \\
\hline PSA (ng/ml) & $7,3 \pm 3$ & $7,5 \pm 2,7$ & 0,64 \\
\hline Vol. Próstata (cc.) & $50 \pm 22$ & $51 \pm 23$ & 0,61 \\
\hline Nódulo ecográfico & $13,4 \%$ & $15,2 \%$ & 0,64 \\
\hline
\end{tabular}




\section{TABLA III. CARACTERÍSTICAS DE LOS PACIENTES CON PRÓSTATAS MENORES O IGUALES DE 50 CC., SEGÚN LA ALEATORIZACIÓN A 6 O 10 BIOPSIAS.}

\begin{tabular}{|l|c|c|c|}
\hline Variable & 6 biopsias & 10 biopsias & $\mathbf{p}$ \\
\hline Edad (años) & $65 \pm 8,8$ & $65,3 \pm 8,6$ & 0,84 \\
\hline PSA (ng/ml) & $7,6 \pm 3,5$ & $7,3 \pm 2,5$ & 0,49 \\
\hline Vol. Próstata (cc.) & $33 \pm 9$ & $36 \pm 9$ & 0,02 \\
\hline Nódulo ecográfico & $19,1 \%$ & $16,9 \%$ & 0,84 \\
\hline
\end{tabular}

En la Tabla I se describen las características de los pacientes.

Al final del estudio $164(52,1 \%)$ pacientes fueron aleatorizados a 6 biopsias y $151(47,9 \%)$ lo fueron a 10 biopsias. En los pacientes asignados a 6 biopsias se cumplió el protocolo en todos los casos, mientras que en los pacientes asignados a 10 biopsias no se pudo completar el protocolo en $4(2,6 \%)$ pacientes. En 3 casos se realizaron 6 cilindros y en uno fueron 7 los cilindros extraídos. Las causas del incumplimiento fueron en 2 casos por dolor, en 1 por lipotimia severa y en el último por negativa del paciente a continuar con el procedimiento.

Al comparar las distintas variables en función de los criterios de asignación en la aleatorización ( ${ }^{\circ}$ de cilindros y volumen prostático), no se encontraron diferencias significativas en la asignación en cuanto al $n^{\circ}$ de cilindros (Tabla II). En el grupo de pacientes con próstatas menores o iguales de $50 \mathrm{cc}$. (Tabla III) se observó una diferencia estadísticamente significativa en el volumen de la próstata $(33 \pm 9 \mathrm{cc}$. frente a $36 \pm 9$ cc., $p=0,02)$. En el grupo de pacientes con próstatas mayores de $50 \mathrm{cc}$. (Tabla IV) se observó una tendencia a la significación en los valores de PSA (7 $\pm 2,2 \mathrm{ng} / \mathrm{ml}$ frente a $7,7 \pm 2,9 \mathrm{ng} / \mathrm{ml}, \mathrm{p}=0,09$ ).

Se detectó cáncer de próstata en $86(27,3 \%)$ pacientes, en la tabla $\mathrm{V}$ se describen las características de los mismos. Se observó una relación estadísticamente significativa entre el diagnóstico de cáncer, la edad ( $p=0,03)$, el volumen prostático $(p=0,0001)$ y la existencia de un nódulo prostático ecográfico $(p=0,0001)$. Así como una tendencia a la significación estadística entre el diagnostico de cáncer y el urólogo que realizó la biopsia $(p=0,1)$. No se observó relación entre el número de biopsias asignadas y el diagnóstico de cáncer $(p=0,37)$, como se observa en la Tabla VI.

Al estratificar por el volumen prostático, observamos que en las glándulas menores o iguales de $50 \mathrm{cc}$ no existen diferencias significativas en el diagnóstico de cáncer en función del número de biopsias realizadas $(p=0,87)$. En cambio cuando la próstata es mayor de $50 \mathrm{cc}$. se diagnosticaron un mayor número de casos de cáncer cuando se realizaron 10 biopsias, con una tendencia a la significación $(p=0,09)$ (Tabla VII).

\section{TABLA IV. CARACTERISTICAS DE LOS PACIENTES CON PRÓSTATAS MAYORES DE 50 CC., SEGÚN LA ALEATORIZACIÓN A 6010 BIOPSIAS.}

\begin{tabular}{|l|c|c|c|}
\hline Variable & 6 biopsias & 10 biopsias & $\mathbf{p}$ \\
\hline Edad (años) & $66,8 \pm 6,4$ & $66,4 \pm 6,8$ & 0,70 \\
\hline PSA (ng/ml) & $7 \pm 2,2$ & $7,7 \pm 2,9$ & 0,09 \\
\hline Vol. Próstata (cc.) & $69,4 \pm 16$ & $72,3 \pm 20$ & 0,33 \\
\hline Nódulo ecográfico & $6,7 \%$ & $12,9 \%$ & 0,25 \\
\hline
\end{tabular}


TABLA V. CARACTERISTICAS PATOLÓGICAS DE LOS PACIENTES CON CÁNCER DE PRÓSTATA.

\begin{tabular}{|c|c|}
\hline Característica & № pacientes (\%) \\
\hline \multicolumn{2}{|l|}{ Score de Gleason: } \\
\hline - No valorable & $1(1,2 \%)$ \\
\hline$\cdot 6$ & $51(59,3 \%)$ \\
\hline$\bullet 7$ & $29(33,7 \%)$ \\
\hline - 8 & $2(2,3 \%)$ \\
\hline - 9 & $2(2,3 \%)$ \\
\hline \multicolumn{2}{|l|}{ Lóbulo afectado: } \\
\hline - Derecho & $31(36 \%)$ \\
\hline - Izquierdo & $30(34,9 \%)$ \\
\hline - Ambos & $25(29,1 \%)$ \\
\hline \multicolumn{2}{|l|}{ Localización: } \\
\hline - Periférico & $63(73,3 \%)$ \\
\hline - Periférico + Medial & $21(24,4 \%)$ \\
\hline - Medial & $2(2,3 \%)$ \\
\hline
\end{tabular}

En $63(73,3 \%)$ pacientes el cáncer se localizó exclusivamente en los cilindros periféricos. En $21(24,4 \%)$ casos se localizó tanto en los cilindros periféricos como en los mediales. Solo en $2(2,3 \%)$ ocasiones el cáncer se localizó exclusivamente en los cilindros mediales, sin que existiera en los periféricos.

Al estratificar estos datos por el volumen de las glándulas, observamos que en las próstatas mayores de $50 \mathrm{cc}$. no se diagnosticó cáncer en los cilindros mediales, como se observa en la Tabla VIII.

En el análisis univariante (regresión logística binaria univariante), la edad mayor de 70 años $(p=0,03)$, el volumen prostático menor de $50 \mathrm{cc}$. $(p=0,001)$ y la existencia del nódulo ecográfico $(p<0,001)$ se asociaron de forma significativa con un incremento en el diagnóstico de cáncer. Existió una tendencia a la significación en función del urólogo que realizó la biopsia $(p=0,1)$ (Tabla IX).

En el análisis multivariante (regresión logística binaria) se introdujeron las variables significativas detectadas en el análisis univariante (la edad, el volumen prostático y la existencia de nódulo eco-

TABLA VI. RELACIÓN ENTRE LAS VARIABLES ESTUDIADAS Y EL DIAGNÓSTICO DE CÁNCER DE PRÓSTATA.

\begin{tabular}{|c|c|c|c|}
\hline Variables & Cáncer $=\mathrm{Si}$ & Cáncer $=$ No & $\mathbf{p}$ \\
\hline $\begin{array}{c}\text { № de biopsias: } \\
\bullet 6 \\
\bullet 10\end{array}$ & $\begin{array}{c}41(25 \%) \\
45(29,8 \%)\end{array}$ & $\begin{array}{c}123(75 \%) \\
106(70,2 \%)\end{array}$ & 0,37 \\
\hline $\begin{array}{c}\text { Volumen próstata (cc.): } \\
\qquad \leq 50 \\
\bullet>50\end{array}$ & $\begin{array}{l}67(37,6 \%) \\
19(13,9 \%) \\
\end{array}$ & $\begin{array}{l}111(62,4 \%) \\
118(86,1 \%) \\
\end{array}$ & 0,0001 \\
\hline $\begin{array}{l}\text { Edad (años): } \\
\qquad \begin{aligned} \bullet & \leq 60 \\
\bullet & 61-70 \\
\bullet & >70\end{aligned}\end{array}$ & $\begin{array}{l}17(21,3 \%) \\
32(23,9 \%) \\
37(36,6 \%) \\
\end{array}$ & $\begin{array}{l}63(78,8 \%) \\
102(76,1) \\
64(63,4 \%) \\
\end{array}$ & 0,03 \\
\hline $\begin{array}{c}\text { Nódulo ecográfico: } \\
\qquad \mathrm{Si} \\
\bullet \text { No }\end{array}$ & $\begin{array}{l}23(51,1 \%) \\
63(23,3 \%)\end{array}$ & $\begin{array}{c}22(48,9 \%) \\
207(76,7 \%)\end{array}$ & 0,0001 \\
\hline $\begin{aligned} & \text { Urólogo: } \\
& \bullet \mathrm{A} \\
& \bullet \mathrm{B} \\
& \bullet \mathrm{C}\end{aligned}$ & $\begin{array}{l}20(29,9 \%) \\
53(30,3 \%) \\
13(17,8 \%)\end{array}$ & $\begin{array}{l}47(70,1 \%) \\
122(69,7 \%) \\
60(82,2 \%)\end{array}$ & 0,1 \\
\hline
\end{tabular}


gráfico). También se introdujo la variable urólogo ya que presentaban una tendencia a la significación. Se identificaron como variables independientes significativamente asociadas al diagnóstico de cáncer, la edad mayor de 70 años $(p=0,005)$, el volumen de la próstata menor de $50 \mathrm{cc}$. ( $p=0,001)$ y la existencia de nódulo ecográfico visible $(p=0,003)$.

Según los datos de nuestra serie se puede calcular la probabilidad de obtener cáncer de próstata en la $1^{\underline{a}}$ biopsia en los pacientes con tacto rectal negativo y PSA entre 3,5 y $20 \mathrm{ng} / \mathrm{ml}$, en función de la edad y del volumen prostático, tal y como se observa en la Tabla XI.

\section{Seguimiento:}

Setenta $(81 \%)$ pacientes diagnosticados de cáncer de próstata fueron susceptibles de tratamiento con intención curativa, $43(61,5 \%)$ fueron tratados con Radioterapia y $27(38,5 \%)$ con Prostatectomía. El score de Gleason de la pieza de prostatectomía fue: 7 en $15(55,5 \%)$ casos, 6 en $9(33 \%), 8$ en $2(7,5 \%)$ y en $1(4 \%)$ paciente el escaso volumen tumoral no permitió su gradación. En $22(81,5 \%)$ pacientes la afectación tumoral fue bilateral (pT2c), en 3 $(11 \%)$ solo unilateral (T2a-b) y en $2(7,5 \%)$ pacientes existía afectación de las vesículas seminales (pT3b). El porcentaje medio de afectación por tumor de la glándula fue de $18 \pm 13$ (1\%-50\%) con una mediana del $10 \%$.

De los 229 pacientes con patología benigna en la biopsia, $116(46,3 \%)$ fueron sometidos al menos a una segunda biopsia durante el tiempo de seguimiento. En las rebiopsias se realizaron entre 8 y 10 cilindros de la zona periférica por paciente. El número medio de biopsias por paciente fue de 1,25 \pm 0,5 (1-3). Al 22,6\% (24 pacientes) se le realizó más de una rebiopsia. En 13 (12,3\%) pacientes se obtuvo

TABLA VII. RELACIÓN ENTRE EL VOLUMEN PROSTÁTICO, EL NÚMERO DE BIOPSIAS Y EL DIAGNÓSTICO DE CÁNCER DE PRÓSTATA.

\begin{tabular}{|c|c|c|c|c|}
\hline & $\leq 50$ cc. & p & $>50$ c. & p \\
\hline № de biopsias: & & 0,87 & & 0,09 \\
\hline$\bullet 6$ & $34(38,2 \%)$ & & $7(9,3 \%)$ & \\
\hline$\bullet 10$ & $33(37,1 \%)$ & & $12(19,4 \%)$ & \\
\hline
\end{tabular}

cáncer de próstata. Las características se resumen en la Tabla XII.

En las glándulas $\leq 50 \mathrm{cc}$., se obtuvo un $18,4 \%$ (9 pacientes) con cáncer, mientras que en las próstata $>50 \mathrm{cc}$ el porcentaje de cáncer fue del $7 \%$ (4 pacientes) $(p=0,07)$.

En los pacientes con próstata $\leq 50 \mathrm{cc}$. $(49$ casos), no se encontraron diferencias significativas en los resultados de la rebiopsia en función del número de cilindros extraídos en la primera biopsia $(22,2 \%$ si 6 cilindros frente a $13,6 \%$ si 10 cilindros, $p=0,34$ ). Cuando la glándula era mayor de $50 \mathrm{cc}$. (57 casos), en los pacientes sometidos a 6 cilindros se encontró un $12,9 \%$ de cáncer frente a ninguno cuando se extrajeron 10 cilindros $(p=0,08)$.

\section{DISCUSIÓN}

En un intento por disminuir el porcentaje de falsos negativos de la biopsia sextante, se han diseñado esquemas de biopsias consistentes en un aumento del número de cilindros extraídos. Estos esquemas de biopsias van desde la extracción de 8 cilindros por paciente, hasta la biopsia por saturación (entre 14 y 45 cilindros) (1 1-16). El diseño de estos esquemas de biopsia se han basado en trabajos clínicos prospectivos bien realizados pero ninguno fue aleatorizado a dos o más esquemas de biopsias.

Diavan et al. (17) diseñaron un esquema de número variable de biopsias para los pacientes con PSA entre 2 y $10 \mathrm{ng} / \mathrm{ml}$, en función de la edad y del volumen prostático, conocido como nomograma de Viena.

En la revisión bibliográfica efectuada solo hemos encontrado 4 estudios prospectivos aleatorizados

\section{TABLA VIII. LOCALIZACIÓN DE LOS CILINDROS CON CÁNCER SEGÚN EL VOLUMEN DE LA PRÓSTATA.}

\begin{tabular}{|l|c|c|c|}
\hline & $\leq 50$ cc. & $>50$ c. & P \\
\hline Localización cáncer: & & & 0,1 \\
\hline - Periférico & $52(72,6 \%)$ & $11(57,9 \%)$ & \\
\hline - Periférico + Medial & $13(19,4 \%)$ & $8(42,1 \%)$ & \\
$\bullet$ Medial & $2(3 \%)$ & & \\
\hline
\end{tabular}


a dos esquemas de biopsia. Tres de ellos mediante biopsia transrectal (18-20) y otro transperineal (21). Los 3 estudios realizados mediante biopsia transrectal tienen importantes defectos metodológicos. Únicamente el de Naughton (18) tiene cálculo del tamaño muestral, pero se incluyen pacientes con y sin tacto rectal sospechoso y, sin y con biopsia previa. En los otros dos estudios se incluyen también pacientes con tacto rectal sospechoso y sin él. En los estudios de Naughton (18) y Kim (19) no se encontraron diferencias significativas entre un esquema de 6 y otro de 12 biopsias $(26 \%-27 \%$, $p=0,9$ y $14,4 \%-17,2 \%$, $\mathrm{p}=0,6$, respectivamente). En el estudio de Paul (20), similar al nuestro (biopsias mediales en el esquema de 10 cilindros), observaron que aunque la ganancia de las biopsias mediales (4\%) en la detección de cáncer de próstata fue baja, era estadísticamente significativa.
En el diseño de nuestro estudio se intentaron evitar los sesgos de inclusión (tacto rectal, biopsias previas, etc.) al imponer unos estrictos criterios de inclusión y se intentó también controlar la variable volumen prostático mediante la doble aleatorización. La proporción del diagnóstico del 0,2 que asumimos al calcular el tamaño muestral, la obtuvimos del histórico de más de 1.000 pacientes biopsiados con estas características. Aunque en el grupo de pacientes con glándulas menores de 50 cc., existía una diferencia estadísticamente significativa del volumen prostático en la asignación a 6 o 10 biopsias (Tabla III) y, una tendencia a la significación estadística en los valores de PSA en el grupo de próstatas mayores de $50 \mathrm{cc}$. (Tabla V), estas diferencias son clínicamente irrelevantes. Por lo tanto, consideramos que no se introdujeron sesgos durante la aleatorización.

TABLA IX. ANÁLISIS UNIVARIANTE DE LAS VARIABLES CLÍNICAS RELACIONADAS CON LA EXISTENCIA DE CÁNCER DE PRÓSTATA.

\begin{tabular}{|c|c|c|c|}
\hline Variable & Riesgo Relativo (HR) & IC95\% & $\mathbf{p}$ \\
\hline Edad (años): & & & 0,03 \\
\hline$\bullet<60$ años & 1,00 & Referencia & \\
\hline - 61 - 70 años & 1,13 & $0,58-2,20$ & 0,7 \\
\hline$\bullet>70$ años & 2,10 & $1,07-4,12$ & 0,03 \\
\hline PSA ng/ml): & & & 0,53 \\
\hline$\bullet \leq 10$ & 1,00 & Referencia & \\
\hline$\bullet>10$ & 1,24 & $0,62-2,46$ & \\
\hline Volumen (cc.): & & & $<0,001$ \\
\hline$\bullet \leq 25$ & 1,000 & Referencia & \\
\hline - 25-50 & 0,55 & $0,23-1,27$ & 0,16 \\
\hline - 51-75 & 0,20 & $0,07-0,51$ & 0,001 \\
\hline$\bullet>75$ & 0,07 & $0,01-0,31$ & $<0,001$ \\
\hline Nódulo: & & & $<0,001$ \\
\hline$\bullet$ No & 1,000 & Referencia & \\
\hline - Si & 3,45 & $1,79-6,57$ & \\
\hline № biopsias: & & & 0,37 \\
\hline$\bullet 6$ & 1,000 & Referencia & \\
\hline$\cdot 10$ & 1,25 & $0,76-2,05$ & \\
\hline Urólogo: & & & 0,10 \\
\hline - A & 1,00 & Referencia & \\
\hline - B & 0,96 & $0,51-1,78$ & 0,90 \\
\hline - $\mathrm{C}$ & 0,46 & $0,20-1,04$ & 0,06 \\
\hline
\end{tabular}



CÁNCER DE PRÓSTATA.

\begin{tabular}{|c|c|c|c|}
\hline Variable & Riesgo Relativo (HR) & IC95\% & $\mathbf{p}$ \\
\hline Edad (años): & & & 0,01 \\
\hline$\bullet<60$ años & 1,00 & Referencia & \\
\hline - 61 - 70 años & 1,55 & $0,74-3,27$ & 0,24 \\
\hline - > 70 años & 2,96 & $1,40-6,28$ & 0,005 \\
\hline Volumen (cc.): & & & $<0,001$ \\
\hline$\bullet \leq 25$ & 13,77 & $3,05-62,02$ & 0,001 \\
\hline - $25-50$ & 8,14 & $2,26-29,22$ & 0,001 \\
\hline - 51-75 & 3,41 & $0,88-13,16$ & 0,07 \\
\hline$\bullet>75$ & 1,00 & Referencia & \\
\hline Nódulo: & & & 0,003 \\
\hline - No & 1,00 & Referencia & \\
\hline - $\mathrm{Si}$ & 3,03 & $1,46-6,28$ & \\
\hline Urólogo: & & & 0,60 \\
\hline - A & 1,00 & Referencia & \\
\hline - B & 0,91 & $0,45-1,82$ & 0,79 \\
\hline - $\mathrm{C}$ & 0,65 & $0,27-1,56$ & 0,33 \\
\hline
\end{tabular}

En nuestro estudio tampoco hemos encontrado diferencias significativas entre ambos esquemas de biopsia en las glándulas menores de 50 cc., aunque existe una tendencia a la significación en las próstatas mayores de $50 \mathrm{cc}$. a favor de la biopsia de 10 cilindros.

TABLA XI. PROBABIILIDAD DE CÁNCER DE PRÓSTATA EN LOS PACIENTES CON TACTO RECTAL NEGATIVO, PSA ENTRE 3,5 Y $20 \mathrm{NG/MLY}$ PRIMERA BIOPSIA EN FUNCIÓN DE LA EDAD Y DEL VOLUMEN PROSTÁTICO.

\begin{tabular}{|c|c|c|c|}
\hline Volumen prostático & \multicolumn{3}{|c|}{ Edad } \\
\cline { 2 - 4 } & $\leq \mathbf{6 0}$ & $\mathbf{6 1 - 7 0}$ & $>\mathbf{7 0}$ \\
\hline $\mathbf{2 5}$ & $30 \%$ & $47 \%$ & $78 \%$ \\
\hline $\mathbf{2 5 - 5 0}$ & $32 \%$ & $34 \%$ & $41 \%$ \\
\hline $\mathbf{5 1 - 7 5}$ & $0 \%$ & $17 \%$ & $28 \%$ \\
\hline $\mathbf{7 7 5}$ & $0 \%$ & $7 \%$ & $15 \%$ \\
\hline
\end{tabular}

En los 3 estudios aleatorizados ya comentados, no se realizó ningún seguimiento de los pacientes sin cáncer de próstata. En nuestro estudio se rebiopsiaron el $46,3 \%$ de los pacientes, detectándose más tumores en las próstata menores de $50 \mathrm{cc}$., aunque sin diferencias significativas en función del número de cilindros de la primera biopsia. En la glándulas mayores de $50 \mathrm{cc}$. se detectaron más tumores con tendencia a la significación, cuando solo se habían practicado 6 cilindros en la primera biopsia.

\section{CONCLUSIÓN}

En las próstatas menores de $50 \mathrm{cc}$. no se encontraron diferencias entre un esquema de 6 y otro de 10 cilindros. Cuando la glándula fue mayor de 50 cc., la tendencia a la significación a favor del esquema de 10 cilindros y la misma tendencia a detectar más tumores en la rebiopsia cuando solo se tomaron 6 cilindros en la $1^{\underline{a}}$ biopsia, nos induce a pensar que un esquema de 6 cilindros puede ser subóptimo en estos casos. Sería necesario la realización de un estudio aleatorizado a 6 - 10 cilindros periféricos (técnica de Stamey) en las próstatas menores de 50 cc para poder evaluar las diferencias entre ambos esquemas. 
TABLA XII. CARACTERISTICAS DE LOS TUMORES DE PRÓSTATA DIAGNOSTICADOS EN LAS REBIOPSIAS.

\begin{tabular}{|l|l|}
\hline Característica & Número (\%) \\
\hline Pacientes con rebiopsia & $116(46,3 \%)$ \\
\hline Cáncer & $13(12,3 \%)$ \\
\hline Afectación tumoral: & $10(77 \%)$ \\
$\bullet$ Un lóbulo & $3(23 \%)$ \\
$\bullet$ Ambos lóbulos & $10(77 \%)$ \\
$\bullet \leq 10 \%$ & $3(23 \%)$ \\
$\bullet>10 \%$ & \\
$\bullet 6$ & $8(61,5 \%)$ \\
$\bullet 7$ & $3(23,1 \%)$ \\
• 9 & $1(7,7 \%)$ \\
\hline Store de Gleason: & $1(7,7 \%)$ \\
\hline
\end{tabular}

\section{BIBLIOGRAFÍA y LECTURAS RECOMENDADAS (*lectura de interés $y^{* *}$ lectura fundamental)}

**1. Heidenreich A, Aus G, Bolla M, Joniau S, Matveev VB, Schmid HP, et al. EAU guidelines on prostate cancer. Eur Urol, 2007; 53:68-80.

*2. Hodge K K, McNeal JE, Terris MK, Stamey .A. Random systematic versus directed ultrasound guided transrectal core biopsies of the prostate. $\mathbf{J}$ Urol, 1989; 142:71-4.

*3. Stamey TA. Making the most out of six systematic sextant biopsies. Urol, 1995; 45:2-12.

4. Levine MA, Ittman M, Melamed J, Lepor H. Two consecutive sets of transrectal ultrasound guided sextant biopsies of the prostate for the detection of prostate cancer. J Urol, 1998; 159:471-5.

5. Stroumbakis N, Cookson MS, ReuterVE, Fair WR. Clinical significance of repeat sextant biopsies in prostate cancer patients. Urology, 1997; 49 (Suppl 3A):113-8.

6. Epstein JI, Walsh PC, Sauvageot J, Carter H B. Use of repeat sextant and transition zone biopsies for assessing extent of prostate cancer. J Urol, 1997; 158:1886-90.

**7. Herranz Amo F, Díez Cordero JM. Cabello Benavente R. Evolución de la técnica de biopsia transrectal ecodirigida de la próstata. Arch.Esp. Urol, 2006; 59:385-396.

8. Uzzo RG, Wei JT, Waldbaum RS, Perlmutter AP, Byrne JG, Vaughan ED Jr. The influence of prostate size on cancer detection. Urol, 1995; 46:831-6.
9. Karakiewicz PI, Bazinet M, Aprikian AG, Trudel C, Aronson S, Nachbe M, et al. Outcome of sextant biopsy according to gland volumen. Urol, 1997; 49:55-9.

10. Letran JL, Meyer GE, Loberiza FR, Brawer MK. The effect of prostate volumen on the yield of needle biopsy. J Urol, 1998; 160:1718-21.

11. Eskew LA, Bare RL, McCullough DL. Systematic 5 region prostate biopsy is superior to sextant method for diagnosis carcinoma of the prostate. $\mathbf{J}$ Urol, 1997; 157:199-202.

12. Babaian RJ, Toi A, Kamoi K, Troncoso P, Sweet J, Evans R, et al. A comparative analysis of sextant and an extended 11-core multisite directed biopsy strategy. J Urol, 2000; 163:152-7.

13. Presti JC, Chang JJ, Bhargava V, Shinohara K. The optimal systematic prostate biopsy scheme should include 8 rather than 6 biopsies: results of a prospective clinical trial. J Urol, 2000; 1636:163.

14. Stewart CS, Leibovich BC, Weaver AL, Lieber MM. Prostate cancer diagnosis using a saturation needle biopsy technique after previous negative sextant biopsies. J Urol, 2001; 166:86-91.

15. de la Taille A, Antiphon P, Salomon L, Cherfan M, Porcher R, Hoznek A, et al. Prospective evaluation of a 21-sample needle biopsy procedure designed to improved the prostate cancer detection rate. Urol, 2003; 61:1181-6.

16. Damiano R, Autorino R, Perdona S, De Sio M, Oliva A, Esposito C, et al. Are extended biopsies really necesary to improve prostate cancer detection?. Prost Cancer Prost Dis. 2003.6:250-5.

*17. Remzi M, Fong YK, Dobrovist M, Anagnostou T, Seitz C, Waldert M, et al. The Vienna nomograms: validation of a novel biopsy strategy defining the optimal number of cores based on patient age and total prostate volume. J Urol, 2005; 174:1256-60.

*18. Naughton CK, Millar DC, Mager DE, Ornstein DK, Catalona WJ. A prospective randomized trial comparing 6 versus 12 prostate biopsy cores: impact on cancer detection. J Urol, 2000; 164:388-392.

*19. Kim JW, Lee HY, Hong SJ, Chung BH. Can a 12 core prostate biopsy increase the detection rate of prostate cancer versus 6 core?: A prospective randomized study in Korea. 2004;45:671-5.

*20. Paul, Schöler S, van Randenborgh H, Kübler H, Alschibaja M, Busch R, et al. Optimization of prostatic biopsy: A prospective randomized trial comparing the sextant biopsy with a 10-core biopsy. Urol Int, 2005; 74:203-8.

21. Emiliozzi P, Scarpone P, DePaula F, Pizzo M, Federico G, Pansadoro A, et al. The incience of prostate cancer in men with prostate specific antigen greater thn $4.0 \mathrm{ng} / \mathrm{ml}$ : a randomized study of 6 versus 12 core transperineal prostate biopsy. J Urol, 2004; 171:197-9. 